\title{
A NOTE ON SALEH'S PAPER 'ALMOST CONTINUITY IMPLIES CLOSURE CONTINUITY’ ${ }^{\dagger}$
}

\author{
by Julian Dontchev and Takashi Noiri
}

(Received 14 August, 1998)

Recently, Saleh [3] claimed to have solved 'a long standing open question' in topology; namely, he proved that every almost continuous function is clousure continuous ( $=\theta=$ continuous). Unforunately, this problem was settled long time ago and even a better result is known. Consider the following implications:

$$
\text { Cont. } \Rightarrow \text { Almost cont. } \Rightarrow \text { Almost } \alpha \text {-cont. } \Rightarrow \eta \text {-cont. } \Rightarrow \theta \text {-cont. } \Rightarrow \text { Weakley cont. }
$$

The first two implications are trivial. In [2], the second author showed that every almost $\alpha$-continuous function is $\eta$-continuous. In [1], Dickman, Porter and Rubin proved that every $\eta$-continuous is $\theta$-continuous and hence weakly continuous.

Although $\theta$-continuous functions behave, in general, nicely, they may cause some unexpected problems. For example, if $f: X \rightarrow Y$ is $\theta$-continuous, then $f: X \rightarrow f(X)$ is not necessarily $\theta$-continuous. Also, the set of all points of continuity of $f: X \rightarrow Y$ may be dense in $X$ and $f$ may not be $\theta$-continuous at any of these points. We show that with the following example.

Example. Consider the classical Dirichlet function $f:\left(\mathbb{R}, \tau_{d}\right) \rightarrow\left(\mathbb{R}, \tau_{d}\right)$, where $\mathbb{R}$ is the real line with the density topology $\tau_{d}$ :

$$
f(x)= \begin{cases}1, & x \in \mathbb{Q} \\ 0, & \text { otherwise }\end{cases}
$$

It is easily observed that $f$ is continuous at every irrational point (furthermore, $\mathbb{P}$, the set of all irrationals is dense in the density topology). On the other hand, $f$ is not weakly $\theta$-continuous at any irrational point.

\section{REFERENCES}

1. R.F. Dickman, Jr., J.R. Porter and L.R. Rubin, Completely regular absolutes and projective objects, Pacific J. Math., 94 (1981), 277-295.

2. T. Noiri, Almost $\alpha$-continuous functions, Kyungpook Math. J., 28 (1988), 71-77.

3. M. Seleh, Almost continuity implies closure continuity, Glasgow Math. J., 40 (1998), 263-264.

\section{Department of Mathematics}

UNIVERSITY OF HELSINKI

PL4, Yliopistonkatu 15

00014 HeLsinki

FinLAND

E-mail: dontchev@cc.helsinki.fi

\author{
Department of Mathematics \\ Yatsushiro College of TeChNology \\ 2627 Hirayama Shinmachi \\ YATSUSHIRO-SHI \\ KUMANMOTO-KEN \\ 866 JAPAN \\ E-mail: noiri@as.yatsushiro-nct.ac.jp
}

${ }^{\dagger}$ This note was written while TAKASHI NOIRI was visiting the Department of Mathematics at University of Helsinki in August 1998. 